\title{
Improved Renal Recovery with Eculizumab Therapy among Children with High Prevalence of Mutation-Associated Atypical Hemolytic Uremic Syndrome: A Retrospective Cohort Study
}

\author{
Abdulaziz A. Bamhraz ${ }^{a, b}$ Khawla A. Rahim ${ }^{a}$ Hassan Y. Faqeehi ${ }^{a}$ \\ Abdulkarim Alanazi ${ }^{a}$ \\ aDivision of Pediatric Nephrology, Children's Specialized Hospital, King Fahad Medical \\ City, Riyadh, Kingdom of Saudi Arabia; ${ }^{b}$ Division of Pediatric Nephrology, Department of \\ Pediatrics, McMaster Children's Hospital, McMaster University, Hamilton, ON, Canada
}

\section{Keywords}

Atypical hemolytic uremic syndrome · Eculizumab · Plasma therapy · End-stage renal disease $\cdot$ Genetic mutations · Consanguineous marriages

\begin{abstract}
Introduction: Genetic defects that determine uncontrolled activation of the alternative complement pathway have been well documented, which account for approximately $40-60 \%$ of atypical hemolytic uremic syndrome (aHUS) cases worldwide. In Saudi Arabia, nearly half of the marriages are consanguineous, resulting in a high prevalence of such genetic diseases. Recent studies have demonstrated the effectiveness of eculizumab against aHUS. Objective: We report our experience of using plasma therapy or/and eculizumab to treat children with aHUS in a tertiary care center in Saudi Arabia and to compare their clinical characteristics, genetic mutations, and treatment outcomes. Methods: A retrospective cohort study was conducted between January 2010 and May 2017. Data, including demographic parameters, clinical presentation, hospital stay duration, need for dialysis, renal recovery, genetic mutations, and outcomes, were obtained from electronic medical records of all eligible patients. Results: Overall, 21 children with aHUS were included, of which 12 (57.1\%) received eculizumab therapy and $9(42.9 \%)$ received only plasma therapy. End-stage renal disease occurred in 7 children (33.3\%), of which $4(57.1 \%)$ received only plasma therapy and $3(42.9 \%)$ received eculizumab therapy whose genetic mutations were not related to the complement dysregulation system. No child who received eculizumab therapy showed recurrence; however, 3 children (33.3\%)
\end{abstract}


who received plasma therapy alone showed recurrence. Genetic mutations were detected in $12 / 20(60 \%)$ of those who underwent genetic screening. Conclusions: Children who received eculizumab therapy showed good renal recovery and maintained remission compared with children who received plasma therapy alone. Genetic mutations were detected in $60 \%$ of the patients, which was associated with a high prevalence of consanguineous marriages.

(C) 2020 The Author(s)

Published by S. Karger AG, Basel

\section{Introduction}

Atypical hemolytic uremic syndrome (aHUS) is a rare disorder caused by overactivation of the alternative pathway of complement and is primarily characterized by thrombotic microangiopathy (TMA) that occurs with a reported incidence of approximately 0.5 per million per year [1-5]. Classic manifestations include thrombocytopenia, microangiopathic hemolytic anemia, and acute kidney injury although other organs are also often affected [6]. In under a decade, complement alternative pathway dysregulation has emerged as the major cause of aHUS, responsible for $60-70 \%$ of cases [4-7]. Various hereditary or acquired deficiencies in the complement alternative pathway proteins have been identified, including (1) inactivating mutations in the genes coding for regulatory proteins of the alternative pathway $\mathrm{C} 3$ convertase (C3bBb), factor $\mathrm{H}(\mathrm{CFH})$, factor I (CFI), membrane cofactor protein (MCP), or thrombomodulin (THBD); (2) anti-CFH antibodies associated with homozygous CFHR1-CFHR3 deletion; and (3) gain-of-function mutations in the genes coding for 2 components of $\mathrm{C} 3 \mathrm{bBb}$ convertase, factor B (CFB) and C3. In addition, common single-nucleotide polymorphisms or haplotypes in the $C F H, C F H R 1$, and $M C P$ genes are susceptibility factors for aHUS [6-8]. In addition, variants of the diacylglycerol kinase $\varepsilon$ (DGKe) gene encode a non-complement-related endothelial cell protein and podocyte protein $[9,10]$.

During the era when plasma infusion (PI) or plasma exchange (PE) was the standard of care by supplying the normal/functional complement inhibitory molecules, they did not address the underlying pathology. The long-and short-term outcome of aHUS patients treated with PE/PI depends on the underlying genetic mutations associated with a poor prognosis prior to the introduction of eculizumab [11]. Historically, $36 \%$ of children and $64 \%$ of adults developed end-stage renal disease (ESRD) or died within 3-5 years of disease onset. Among children, $57 \%$ relapse occurred during the first year of the disease $[6,12]$.

Eculizumab (Soliris ${ }^{\circledR}$, Alexion Pharmaceuticals, Inc., Boston, MA, USA) is a humanized monoclonal complement inhibitor that is the first and the only approved treatment for patients with aHUS $[13,14]$. Eculizumab binds with high affinity to C5, inhibiting C5 cleavage into $\mathrm{C} 5 \mathrm{a}$ and $\mathrm{C} 5 \mathrm{~b}$ and preventing the generation of the terminal complement complex C5b-9, thus inhibiting complement-mediated TMA. Eculizumab was proven to be effective in patients with aHUS in 5 clinical studies [15-18], in which it resolved and prevented complementmediated TMA, improving renal function and hematologic outcomes [3]. In a recent review, Wong et al. [19] have reported that eculizumab therapy was effective in approximately $85 \%$ of the patients who were resistant to or dependent on plasma therapy.

However, no such studies regarding aHUS have been reported in the Middle East. The present study aimed to report our experience of using plasma therapy or eculizumab for treating children with aHUS in a tertiary care center in Saudi Arabia and to compare their clinical characteristics, genetic mutations, and treatment outcomes between therapies. The present study may represent an initiative for the establishment of an aHUS patient registry in the Middle East, providing opportunities for successful multicentric collaborations and partnerships as well as advancement of scientific research in this region. 


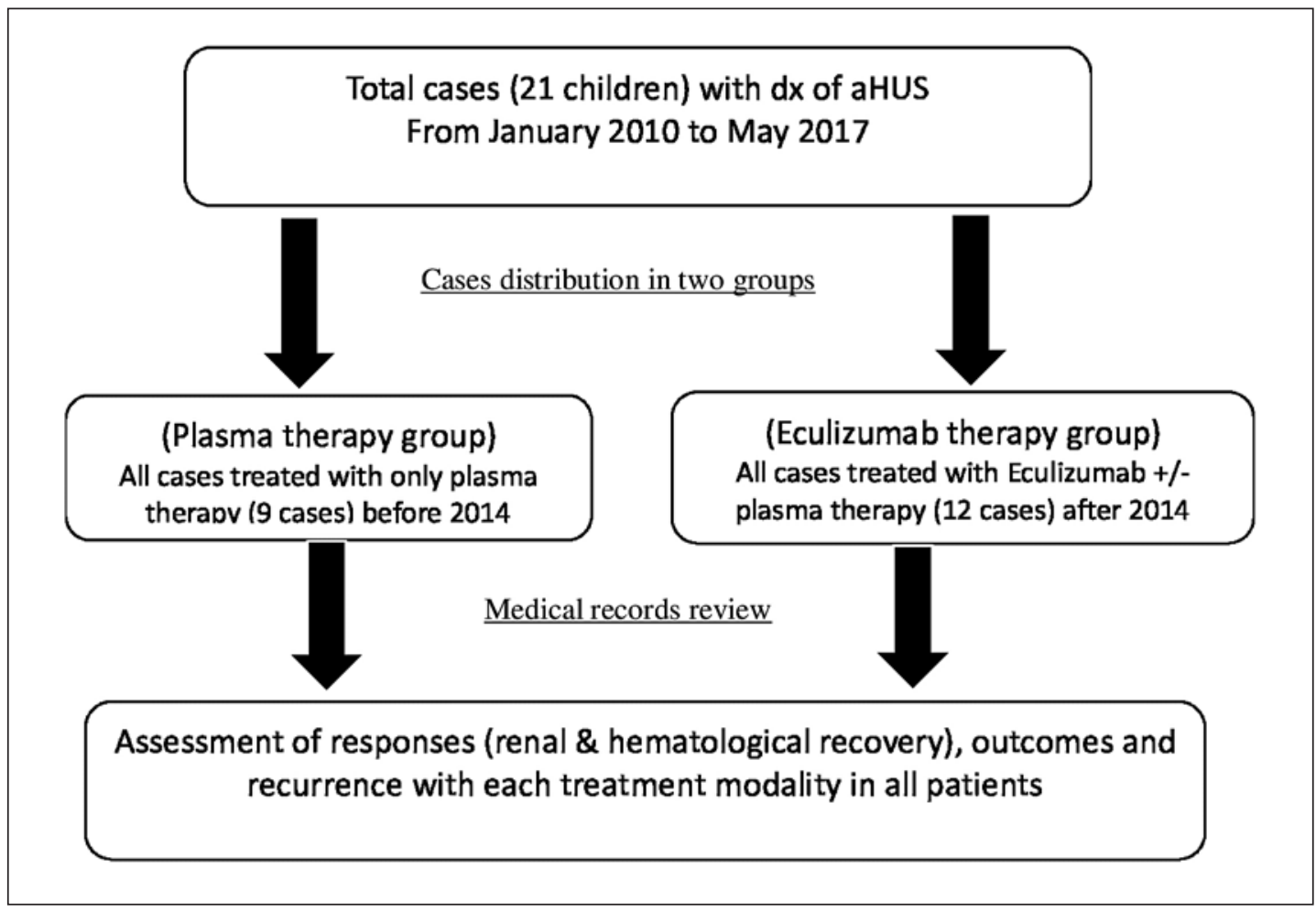

Fig. 1. Flow diagram of case distribution. aHUS, atypical hemolytic uremic syndrome.

\section{Materials and Methods}

The present single-center retrospective cohort study was conducted at the department of pediatric nephrology at King Fahad Medical City. Data of all children diagnosed with aHUS who presented with hematological involvements (anemia, low platelet count, high LDH, schistocytes, and low haptoglobin with normal ADAMT13 activity) and either renal involvement (acute kidney injury [AKI] and renal biopsy finding of TMA), excluding all cases of secondary HUS, were collected. Thus, we excluded patients with HUS secondary to drugs, autoimmune diseases, or infections (caused by Shiga toxin-producing Escherichia coli, Streptococcus pneumoniae, or HIV), bone marrow or solid organ transplantation, and cobalamin deficiency who were admitted between January 2010 and May 2017. All data were retrieved from electronic medical records.

The data retrieved included information regarding age, sex, presenting symptoms, diarrhea presence or absence, hospital stay duration, laboratory data on admission and at follow-up, blood and plasma transfusions, need for dialysis, response to PE alone and/or eculizumab, and patient outcomes as mentioned in Figure 1. All children with aHUS who received either PE or eculizumab were assessed, and missing data were retrieved by reviewing patient medical records from other sources, such as computer systems and paper records, or by contacting external laboratories that performed the complement assays and genetic studies via E-mail.

Stool culture for Shiga toxin-producing organisms was performed in all patients who presented with diarrhea. Proteinuria was assessed using a urine dipstick or spot urine test for protein/creatinine ratio on admission and at follow-up. Blood samples were collected and stored before the initiation of plasma therapy for all children after 2014 and all those diagnosed before that if still on regular follow-up in our center as mentioned in Appendix 1. 
Kidney and

Blood Pressure

Research

Table 1. Patient characteristics at onset

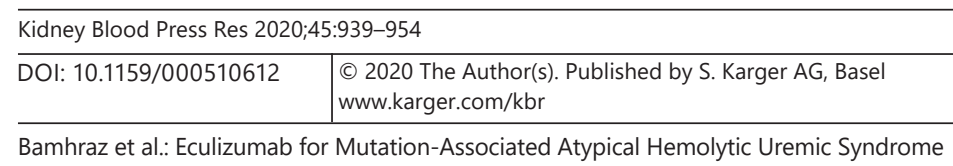

\begin{tabular}{|c|c|c|}
\hline Characteristic & Result $(n=21)$ & $\%$ \\
\hline Male/female & $16 / 5$ & \\
\hline Age at onset, years, mean (range) & $3.9(0.1-11.5)$ & \\
\hline Familial HUS history & 6 & 28.5 \\
\hline Parental consanguinity & 15 & 71.4 \\
\hline Triggering events & 13 & 61.9 \\
\hline URTI & 9 & 42.8 \\
\hline GE & 3 & 14.3 \\
\hline Trauma & 1 & 4.8 \\
\hline Critical care admission at onset & 14 & 66.7 \\
\hline \multicolumn{3}{|l|}{ Extra-renal involvement } \\
\hline Hepatitis & 7 & 33 \\
\hline Neurologic involvement & 6 & 28.5 \\
\hline Intra-alveolar hemorrhage/respiratory failure & 5 & 23.8 \\
\hline Others* & 2 & 9.4 \\
\hline Oliguria/anuria & 16 & 76.2 \\
\hline HTN at onset & 18 & 85.7 \\
\hline Mean hemoglobin, g/dL & $8.8(5.1-13.3)$ & \\
\hline Mean serum creatinine, $\mu \mathrm{mol} / \mathrm{L}$ & $302(24-820)$ & \\
\hline Dialysis required & 16 & 76.1 \\
\hline \multicolumn{3}{|l|}{ Platelet count } \\
\hline$>150 \times 10^{9} / \mathrm{L}$ & 4 & 19.1 \\
\hline $100-150 \times 10^{9} / \mathrm{L}$ & 3 & 14.3 \\
\hline $50-99 \times 10^{9} / \mathrm{L}$ & 7 & 33.3 \\
\hline$<50 \times 10^{9} / \mathrm{L}$ & 7 & 33.3 \\
\hline Complete triad of conditions** & 12 & 57.1 \\
\hline Low C3 & 14 & 66.7 \\
\hline
\end{tabular}

HUS, hemolytic uremic syndrome; URTI, upper respiratory tract infection; GE, gastroenteritis; HTN, hypertension. * Others: pericarditis in 1 case and multiorgan failure in 1 case ( $4.7 \%$ each). ** Complete triad: hemoglobin $<10 \mathrm{~g} / \mathrm{dL}$, platelet count $<150 \times 10^{9}$ cells $/ \mathrm{L}$, and serum creatinine above the upper limit of the normal range.

In all children whose parents provided consent, genetic screening for aHUS was performed by direct sequencing of the coding sequences and flanking intronic lesions. Genomic DNA was extracted from blood leukocytes using a next-generation sequencing panel in accredited genetic laboratories as mentioned in Appendix 1 for all known aHUS genes, including those encoding CFH, CFHRs, CFI, CFB, C3, MCP, THBD, and DGKe. Then, whole exome sequencing for those with negative genetic panel and segregation analysis for each variant in those with positive results were performed.

Plasma therapy as a standard treatment was compared with eculizumab therapy. Up to 2014, patients were treated with the standard therapy involving $20 \mathrm{~mL} / \mathrm{kg}$ fresh frozen plasma twice per day or PE (1.5 $\times$ plasma volume) for 5-10 daily sessions until renal and hematological responses were achieved. Then, patients were weaned gradually by continuing the treatment every other day along with response monitoring and subsequently maintained on fresh frozen plasma therapy once weekly. After 2014, all patients who were suspected or confirmed of aHUS, after taking the required blood tests as stated above, received a quadrivalent meningococcal conjugate vaccine (Men-CACYW-135), Menactra ${ }^{\circledR}$, with booster dose after 4-6 weeks later and started on (penicillin V) prophylaxis dose appropriate for age before giving the recommended doses of eculizumab for induction and main- 
Kidney and

Blood Pressure

Research

Table 2. Genetic mutations in children with aHUS

Table 3. Identified genetic mutations in children with aHUS

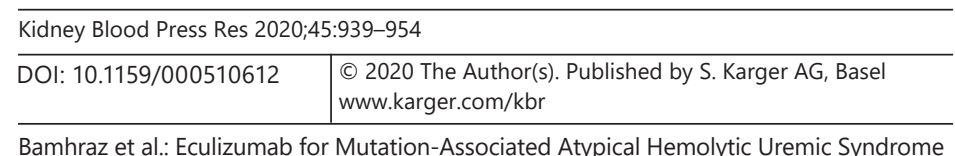

aHUS, atypical hemolytic uremic syndrome.

\begin{tabular}{lll}
\hline Identified genetic mutations & $n=12 / 20$ & $60 \%$ \\
\hline Complement associated & 7 & 35 \\
DGKe & 2 & 10 \\
Others* & 3 & 15 \\
\hline
\end{tabular}

aHUS, atypical hemolytic uremic syndrome. * Other mutations were discovered in 3 patients ( 1 child each, harboring a mutation in the WT1, $L A M B 2$, or MCCC2 gene).

tenance based on body weight according to the eculizumab protocol for aHUS treatment as in Appendix 2.

Sample size was based on the number of patients meeting the inclusion and exclusion criteria during the study period between 2010 and 2017. Patients were stratified according to the available therapy type before and after 2014. Analyses were based on nonparametric statistical methods owing to the small sample size. The 2 therapies were compared using Fisher's exact test. In addition, the group that received eculizumab therapy and maintained remission with this therapy was followed up to determine the average percentage of improvement from the initiation of the therapy until the last follow-up using the Wilcoxon matched-pair test. Side effects of eculizumab therapy were monitored in such patients who received this therapy during the long-term follow-up. Data were analyzed using SPSS software version 21 (IBM Corp., Armonk, NY, USA), and $p$ value of $<0.05$ was considered significant.

\section{Results}

A total of 21 children met the study criteria. Most of the patients (16 [76\%]) were from the central and northern regions of Saudi Arabia (8 patients from each region). Patient characteristics at aHUS onset are summarized in Table 1. Mean age at onset was 3.9 years, with 9 children (43\%) experiencing disease onset before 2 years of age. Male-to-female ratio was 3:1. Upper respiratory tract infection (URTI) was the most frequent concomitant factor ( 9 patients [42.8\%]). The most frequent extra-renal manifestation was hepatitis (7 patients [33\%]), followed by neurological manifestations (altered consciousness or coma, seizures, and focal neurological deficits in 6 patients [28.5\%]).

\section{Genetic Mutations}

The 21 patients included in the study were from 19 unrelated families. Familial aHUS was noted in 6 of the 19 families (31.5\%). Genetic studies were performed in almost all cases (20/21), and mutations were detected in 12 of the 20 children (60\%). Among these 12 cases, mutations in complement-related genes were detected in 7 (35\%) as summarized in Tables 
Kidney and
Blood Pressure

Research
Kidney Blood Press Res 2020;45:939-954 DOI: $10.1159 / 000510612$

(c) 2020 The Author(s). Published by S. Karger AG, Basel www.karger.com/kbr

Bamhraz et al.: Eculizumab for Mutation-Associated Atypical Hemolytic Uremic Syndrome

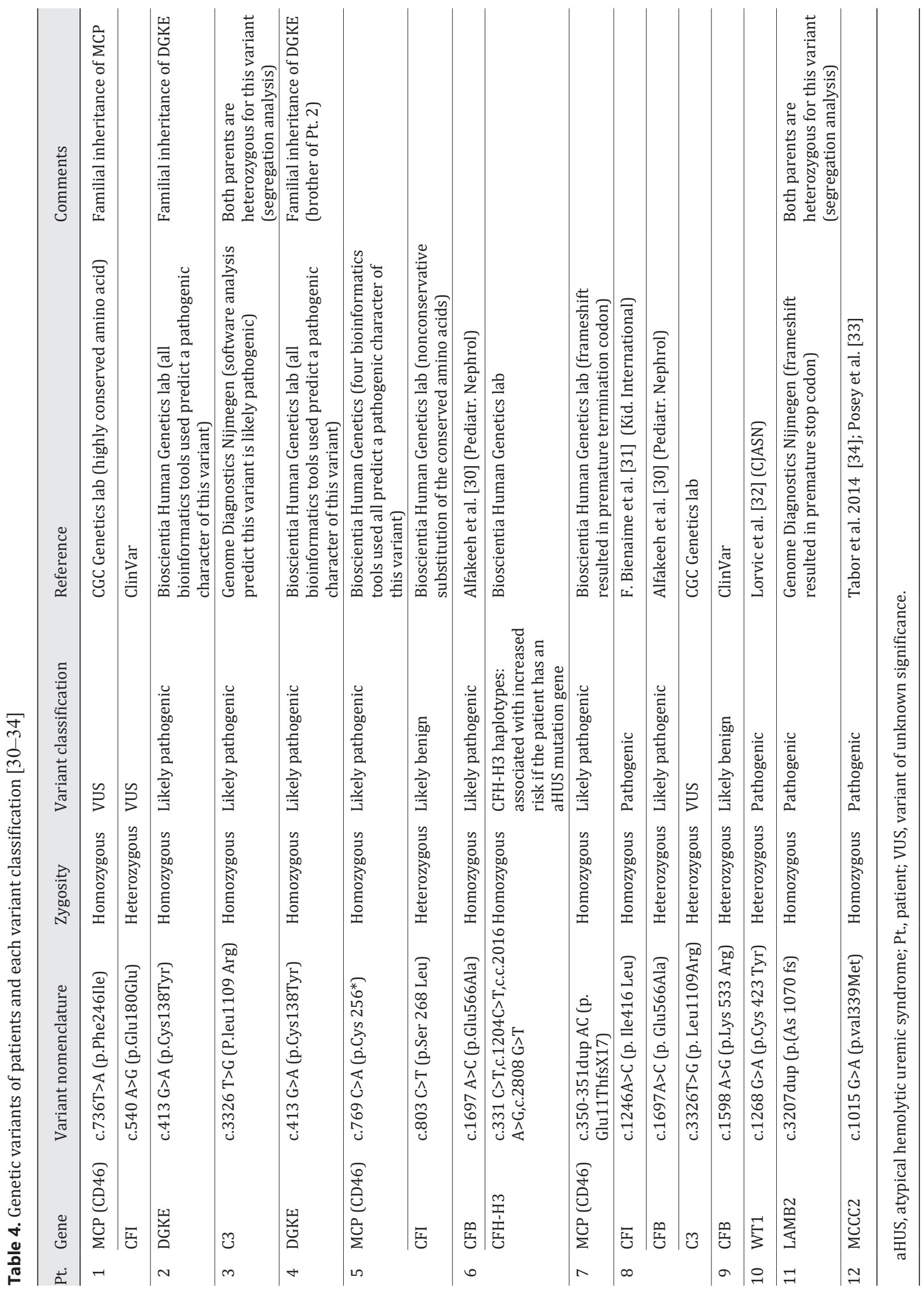




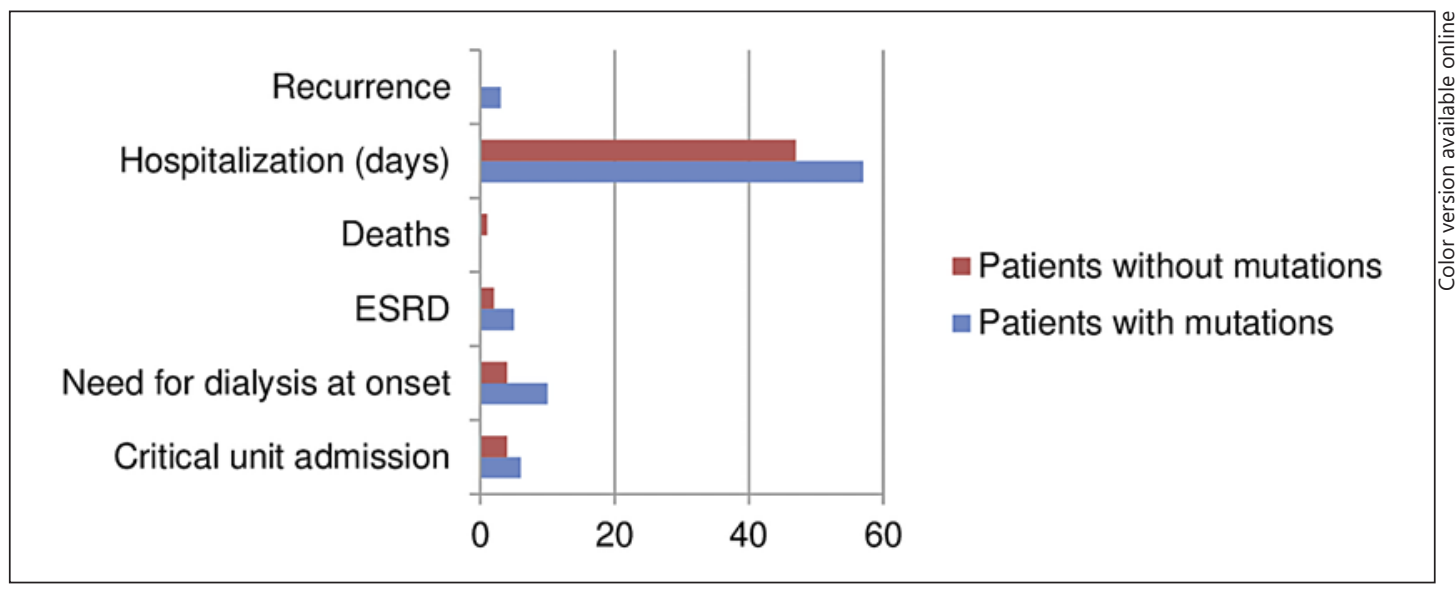

Fig. 2. Genotype-phenotype correlations. Comparison of disease severity between children with aHUS with and without genetic mutations according to critical care admission, dialysis requirement, disease recurrence, ESRD evolution, and death. aHUS, atypical hemolytic uremic syndrome; ESRD, end-stage renal disease.

2 and 3. Combined mutations ( $>1$ mutation either digenic or more) were detected in 4 families. There were 2 cases of homozygous MCP with heterozygous CFI, 1 case of homozygous CFB and CFH haplotypes, and 1 case of homozygous CFI with heterozygous C 3 and CFB mutations. However, there were 3 cases with single-gene mutation. None of the patients presented with THBD or CFHR and anti-CFH autoantibodies. Non-complement-related mutations were noted in 5 cases, 2 cases had DGKe homozygous mutation within same family siblings, and 3 cases had mutation in the WT1, LAMB2, and MCCC2 genes, with one in each case. Genetic mutation variants discovered in our patients are presented in Table 4. Children with non-complementassociated genetic mutations who presented with clinical features of aHUS received plasma therapy, followed by eculizumab therapy, to maintain remission until the genetic results were obtained; then, the treatment was discontinued.

Correlations between Genetic Mutations, Clinical Phenotype, and Response to Therapy

Patients with genetic mutations were younger, had a more severe disease course, required more frequent dialysis sessions, and primarily experienced ESRD with a longer hospital stay than did those without mutations (Fig. 2; Table 5). The correlation between the genetic mutations and response to therapy is mentioned in Table 6.

\section{Therapy Groups}

Patient outcomes after initiating therapy are presented in Table 7. A total of 9 patients were treated with only plasma therapy before 2014, of which 5 (55\%) showed complete renal recovery, whereas the remaining 4 (45\%) experienced ESRD. A total of 12 patients were treated with eculizumab with or without plasma therapy, of which 9 (75\%) showed complete renal recovery without recurrence. Overall, 3 patients with non-complement-associated mutations experienced ESRD with eculizumab therapy, while 1 without mutation died from secondary hemophagocytic lymphohistiocytosis, as identified via bone marrow biopsy. Interestingly, 1 patient with partial aHUS (only renal involvement with normal hematological parameters) which proved to have TMA on renal biopsy and positive genetic study in complement-related genes (Pt. 8 in Table 4) who was on dialysis for over 8 months recovered completely after initiating eculizumab therapy. In those who had recovery with plasma therapy, after discontinuation of plasma therapy, recurrence occurred in 3 patients, 1 of 
Table 5. Correlations between genetic mutations and clinical phenotype

\begin{tabular}{lccc}
\hline & Patients with mutations & Patients without mutations & $p$ value \\
\hline Age at onset, years, mean (range) & $2.8(0.1-10)$ & $5.8(0.4-11.5)$ & 0.12 \\
Critical unit admissions & 6 & 4 & 0.58 \\
Need for dialysis at onset & 10 & 4 & 1.00 \\
ESRD & 5 & 2 & 1.00 \\
Death & 0 & 1 & 0.31 \\
Hospitalization, days, mean (range) & $57(7-127)$ & $47(23-125)$ & 0.591 \\
Recurrence & 3 & 0 & 0.20 \\
\hline
\end{tabular}

ESRD, end-stage renal disease.

which achieved remission with plasma therapy, whereas 2 achieved remission after switching to eculizumab therapy. Hospital stay was 1 week longer in the plasma therapy group than in the eculizumab therapy group.

\section{Long-Term Follow-Up of Patients Receiving Eculizumab Therapy}

Table 8 presents the average percentage improvement in 8 of the 9 patients who received eculizumab therapy (1 patient was lost long-term follow-up at the time of data collection). Median follow-up period after the initiation of eculizumab therapy was 23 (range: 3-35) months. Of the 8 patients, 7 achieved complete renal and hematologic recovery $(p=0.036$ and $p=0.012$, respectively). Only 1 patient experienced partial renal recovery and continued to show a low platelet count with a prolonged coagulation profile.

\section{Common Side Effects of Eculizumab Therapy}

Metabolic acidosis was the most frequent side effect of eculizumab therapy (4 patients [72\%]) (Fig. 3).

\section{Discussion}

We have conducted the largest retrospective cohort study of children with aHUS in the Arab region so far. aHUS can occur at any age (from the neonatal period to old age), with extremes at 1 day of age and 83 years of age $[7,20]$. Reportedly, $70 \%$ of the children experience the first episode of the disease before 2 years of age, and approximately $25 \%$ of these children experience the first episode before 6 months of age; moreover, childhood onset aHUS is equally frequent in boys and girls [20].

Baskin et al. [12] have reported that the median age at aHUS onset was 4 years, which is similar to the age noted in the present study (mean age at onset: 3.9 years). In the present study, $43 \%$ of the children with aHUS were below 2 years of age, which is lower than the rate of 56\% reported by Fremeaux-Bacchi et al. [6] which might be attributed to small-sample single-center data.

Furthermore, in the present study, male-to-female ratio was 3.2, which is higher than the ratio of 2 and 1.1 reported by Fujisawa et al. [21] (Japanese cohort) and Fremeaux-Bacchi et al. [6] (French series), respectively, and this supports the global aHUS registry data that males have higher incidence during childhood than females as reported by Schaefer et al. [22].

Other infections, particularly URTI and diarrhea/gastroenteritis, have been reported to trigger aHUS in at least $50 \%$ of the patients [7] and up to $80 \%$ of the cases in some pediatric 
Kidney and
Blood Pressure

Research

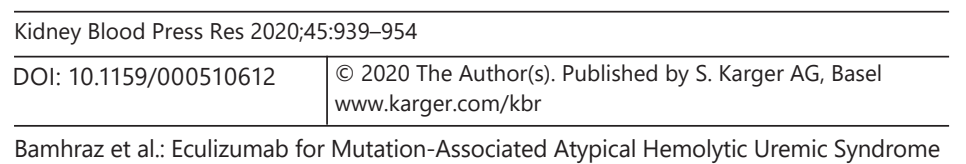

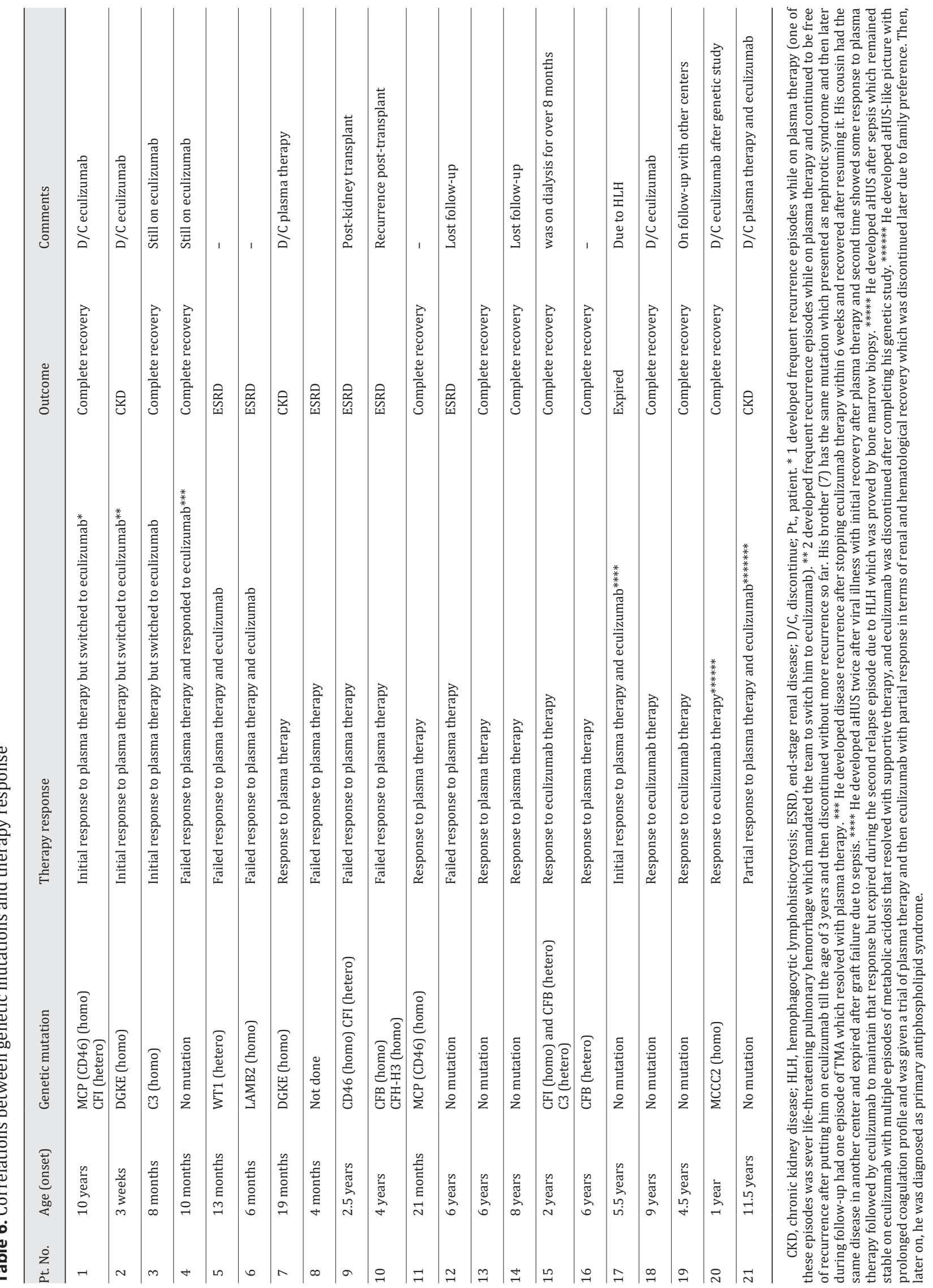


Kidney and

Blood Pressure

Research

\begin{tabular}{l|l}
\hline Kidney Blood Press Res 2020;45:939-954 \\
\hline DOI: 10.1159/000510612 & $\begin{array}{l}\text { @ 2020 The Author(s). Published by S. Karger AG, Basel } \\
\text { www.karger.com/kbr }\end{array}$ \\
\hline
\end{tabular}

Bamhraz et al.: Eculizumab for Mutation-Associated Atypical Hemolytic Uremic Syndrome
Table 7. Patient outcomes after starting therapy

\begin{tabular}{lcl}
\hline & $\begin{array}{l}\text { Plasma group } \\
\text { (9 cases) }\end{array}$ & $\begin{array}{l}\text { Eculizumab ( } \pm \text { plasma) } \\
\text { group (12 cases) }\end{array}$ \\
\hline Renal recovery & 5 & 9 \\
ESRD & 4 & 2 \\
Death & 0 & 1 \\
Mean hospital stay, days & 56.2 & 49.9 \\
\hline
\end{tabular}

ESRD, end-stage renal disease.

Table 8. Paired comparisons via the Wilcoxon matched-pair test at the therapy initiation and last follow-up

\begin{tabular}{|c|c|c|c|c|c|}
\hline & \multicolumn{2}{|l|}{ At therapy initiation } & \multicolumn{2}{|c|}{ At the last follow-up } & \multirow{2}{*}{$\begin{array}{l}p \text { value } \\
\text { (Wilcoxon test) }\end{array}$} \\
\hline & mean \pm SD & median (min, max) & mean \pm SD & median (min, max) & \\
\hline Creatinine, $\mu \mathrm{mol} / \mathrm{L}$ & $140.5 \pm 158.6$ & $78(19,504)$ & $42.8 \pm 15.8$ & $39(20,68)$ & 0.036 \\
\hline Hemoglobin, g/dL & $9.4 \pm 1.3$ & $9.1(8,11.9)$ & $12.4 \pm 1.7$ & $12.7(10.1,14.6)$ & 0.012 \\
\hline Platelet count, $10^{9} / \mathrm{L}$ & $239.8 \pm 149.7$ & $201(87,488)$ & $266.0 \pm 82.0$ & $255(110,354)$ & 0.779 \\
\hline $\mathrm{LDH}, \mathrm{U} / \mathrm{L}$ & $356.1 \pm 104.7$ & $322.5(236,540)$ & $317.3 \pm 117.1$ & $279.5(185,561)$ & 0.441 \\
\hline C3 (0.9-1.8) & $0.85 \pm 0.55$ & $1(0.04,1.6)$ & $1.01 \pm 0.48$ & $0.90(0.6,2.0)$ & 0.499 \\
\hline \multirow[t]{2}{*}{ Final status } & Complete recovery & $7(87.5 \%)$ & & & \\
\hline & Partial recovery & $1(12.5 \%)$ & & & \\
\hline
\end{tabular}

SD, standard deviation; min, minimum; max, maximum; LDH, lactate dehydrogenase.

Fig. 3. Side effects of eculizumab therapy in children with aHUS. Our study population showed the following prevalence of side effects: metabolic acidosis, 72\%; drug allergy reaction, 14\%; hepatic side effects, $14 \%$; and sepsis and meningitis, $0 \%$. aHUS, atypical hemolytic uremic syndrome.

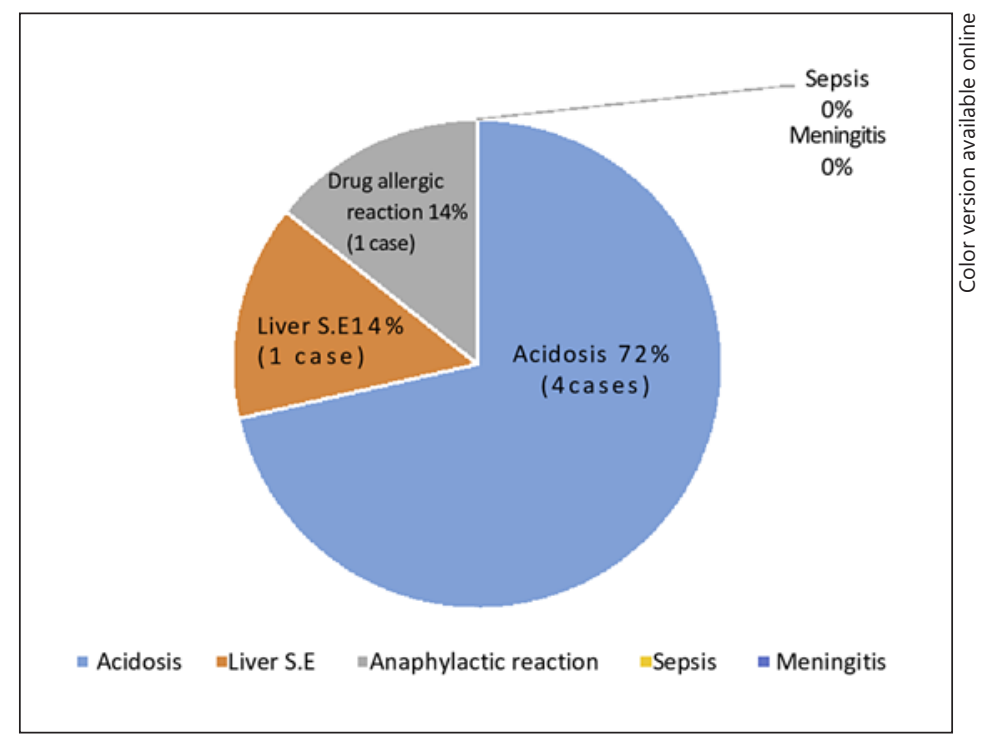

cohorts [16, 17]. Noris et al. [7] have reported that $70 \%$ of the patients presented with diarrhea and/or gastroenteritis and URTI as frequent triggers, which is similar to the finding of the present study (62\%), with URTI being the major precipitating factor (42.8\%).

While decline in kidney function is usually a most common aHUS manifestation at presentation, an estimated 10-48\% of aHUS patients may experience extra-renal manifestations 
with cardiovascular, pulmonary, gastrointestinal, or varied loss of functions in other organs or systems $[11,20]$. Pancreatitis, intestinal bleeding, and hepatic cytolysis have been reported in $5 \%$ of the patients with aHUS $[7,20]$. Similarly, in the present study, hepatitis (increase in hepatic enzyme levels) was the most frequent (33\%) extra-renal involvement. FremeauxBacchi et al. [6] have demonstrated neurologic involvement in $16 \%$ of the patients with aHUS, whereas in the present study, we demonstrated neurologic complications in $28.5 \%$ of the patients. Such a higher percentage of extra-renal manifestations in the present study may be attributed to disease severity at presentation. Furthermore, Fremeaux-Bacchi et al. [6] have reported that $59 \%$ of the children showed severe renal involvement at disease onset; however, renal involvement was more severe in the present study, with $76.1 \%$ of the patients requiring dialysis at the first aHUS episode and $66.7 \%$ requiring admission to critical care unit. In addition, $66.7 \%$ of the patients in the present study exhibited low C3 levels, which is higher than the rate of $50 \%$ reported by Baskin et al. [12].

Genetic or acquired dysregulation of the complement alternative pathway is detected in $40-60 \%$ of patients with aHUS suggesting a genetic predisposition $[6,7,23]$. In the present study, $35 \%$ of the patients harbored mutations in the complement genes, resulting in the loss of endothelial cell protection from complement attack and ultimately leading to TMA lesions, which is higher than that reported by Lee et al. [24] (Korean pediatric series) but lower than the recent findings of the largest prospective cohort study of eculizumab in aHUS patients by Menne et al. [3]. However, our results are highly significant if we consider that the study had been done only in 1 center within Saudi Arabia.

Familial aHUS in which 2 or more persons develop aHUS at different times without exposure to common triggering infectious agents or when disease-causing mutations are identified in one of the genes was reported in $20 \%$ of aHUS cases by Bu et al. [25]. In our study, familial aHUS was reported in 6 of 19 families (35.1\%) which might be attributed to the high prevalence of consanguinity in the Saudi population, with $71.4 \%$ of our cases showing parental consanguinity.

In the present study, $15 \%$ of the patients harbored mutations in the $C F I, M C P$, or $C F B$ genes, with $5 \%$ harboring mutations in $\mathrm{CFH}$ and $10 \%$ in $\mathrm{C3}$; these rates are lower than those reported by Bresin et al. [26] (22.6 and $27 \%$ of the patients with mutations in the MCP and $C F I$ genes, respectively, and 8-10\% with mutations in the $C F H, C 3$, or $C F B$ genes). Combined mutations were frequent and detected in $20 \%$ of patients in our study which is higher than that reported in 4 independent cohorts comprising the International Registry as well as French, Spanish, and UK cohorts; there were only 3.4\% combined mutations among 795 patients with aHUS (including probands and affected relatives) [7, 26]. In addition, patients with underlying mutations, particularly combined mutations, presented with more severe disease, like patient 8 in Table 4 who was on dialysis over 8 months and then recovered after starting eculizumab therapy as reported from our center by Alzabli et al. [27], than did those with single or no mutations. This finding is similar to that of a study by Bresin et al. [26] who reported that ESRD developed within 3 years of onset in 50\% of CFH or CFI patients with combined MCP mutations and 19\% of patients with an isolated MCP mutation. In the present study, 2 siblings from the same family (patients 2 and 4 in Table 4) showed homozygous DGKe gene mutations with frequent relapse episodes before 5 years of age. Chronic kidney disease developed even with the use of eculizumab in one of those patients, but the disease pattern did not change. This finding is supported by a review article by Lemaire et al. [10] stating that the absence of evidence linking DGKe deficiency to the complement cascade and relapse of acute HUS in patients receiving eculizumab therapy indicate no benefits among such patients.

Identification of mutations can help confirm the diagnosis of complement-mediated disease, and genetic counseling and prognosis assessment can potentially determine treatment duration and treatment choice for preventing post-kidney transplant recurrence.

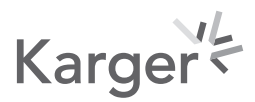


Historically, aHUS was managed with PE/PI and was associated with high morbidity and mortality rates $[6,12,18]$. Baskin et al. [12] have reported that recovery of clinical and laboratory parameters was achieved in $50 \%$ of the patients receiving plasma therapy, but relapse occurred after therapy discontinuation, which is similar to the results of the present study showing that $55 \%$ of the patients achieved renal recovery, $33.3 \%$ experienced recurrence, and $45 \%$ developed ESRD. These data might not reflect the actual recurrence rate with this therapy in our cohort as some patients presented with ESRD to start with, some were lost to follow-up in our center, and others switched to the other therapy group as the above patients.

Eculizumab has profoundly changed aHUS management, and it is currently approved for adult and pediatric patients $[3,28]$. In previous prospective studies, aTMA event-free status and complete TMA response were noted in $>92 \%$ with eculizumab and $50-85 \%$ of aHUS patients who were plasma therapy resistant and plasma therapy dependent, respectively [17, 29]. After the exclusion of STEC-HUS and ADAMT13 deficiency, eculizumab therapy can be the first-line treatment for aHUS, if available. In the present study, $75 \%$ of the patients who received eculizumab therapy achieved complete recovery over a short hospital stay and showed no recurrence, whereas $25 \%$ of the patients developed ESRD; these patients harbored mutations in the non-complement-related genes, which may explain the failure of eculizumab therapy. Compared with those who received plasma therapy, the recovery rate was higher and no recurrence was observed in patients who received eculizumab although these differences were not statistically significant, probably because of the small sample size.

Eculizumab is well tolerated, but bone marrow failure, wrist fracture, and acute respiratory failure have been reported as unrelated severe adverse events [18]. Recently, liver toxicity associated with eculizumab was reported in a few patients with aHUS [28]. In the present study, elevated liver enzyme levels were noted in some patients before and after receiving eculizumab. Overall, there was a strong trend toward normalization of hepatic enzyme levels after eculizumab initiation. Our findings suggest that increased alanine aminotransferase and aspartate aminotransferase levels noted in some patients may be related to pre-existing, ongoing TMA that developed prior to treatment initiation which was similar to what has been reported by Greenbaum et al. [18]. Additionally, metabolic acidosis was observed in $72 \%$ of our patients. Fortunately, none of these patients presented with meningitis or sepsis as all of them received meningococcal vaccination and prophylactic antibiotics prior to the initiation of eculizumab therapy.

The present study has some limitations. First, the results of this study are partially comparable with the results of other studies, and this might be attributable to its small sample size. This limits the extent and accuracy of comparisons. Second, long-term follow-up was only reported for patients who received eculizumab therapy because a well-organized aHUS registry was established in our center at the time of introduction of eculizumab therapy. Third, details of aHUS patients who received plasma therapy were not recorded systematically in the hospital database. Hence, some patients had missing data, which needed to be obtained by contacting the attending physicians and the laboratory department. We are building our aHUS registry including the data obtained from all patients since diagnosis and follow-up, which will support further studies in the future.

\section{Conclusions}

Compared with plasma therapy, eculizumab therapy maintained remission and was associated with favorable renal recovery. The number of complement-associated mutations detected in our study cohort was quiet significant keeping in mind the single-center data in one of the countries with highest inherited disease due to high consanguineous marriages.

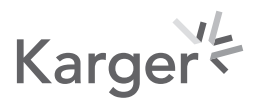


Currently, eculizumab therapy can be considered a life-saving therapy, and it may improve patients' quality of life. However, information regarding the optimal duration of treatment, safe time to discontinue eculizumab, and patients at a high risk of severe recurrence remains limited. Future collaborative studies are warranted to clarify these aspects in this region of the world.

\section{Acknowledgements}

We would like to thank our HUS program coordinator Ms. Anita Varghese for her assistance in data collection; our medical secretary Ms. Norma Parogan for editing; pediatric medical genetic section help especially Dr. Mohammad Balawi and Dr. Eissa Faqeehi for valuable review of our patient cohort genetic results with extensive support in reviewing the variants' nomenclature; the research center, particularly Dr. Tariq Wani, for his help in statistical analysis; Dr. Humairiah for reviewing this manuscript, and the Scientific Writing Department at King Fahad Medical City for their help in manuscript writing. Moreover, we would like to thank the King Fahad Medical City-Research Center for funding our manuscript writing and editing costs.

\section{Statement of Ethics}

The study methodology has been approved by the Institute Research Board (IRB) committee on human research with IRB Log No. 17-091. A written consent has been received from the patients' parent. The study was performed according to the principles of the Declaration of Helsinki.

\section{Conflict of Interest Statement}

The authors declare that they have no conflicts of interest.

\section{Funding Sources}

King Fahad Medical City-Research Center funded the research manuscript writing and editing costs.

\section{Author Contributions}

A. Bamhraz carried out the data collection, data analysis, and manuscript writing. $\mathrm{K}$. Rahim carried out manuscript review, data interpretation, and manuscript writing with proper organization. H. Faqeehi and A. Alanazi reviewed the manuscript. 


\section{Appendix 1. aHUS Program Laboratory Tests}

aHUS program laboratory investigations

\section{A. Initial laboratory investigation(s) (for all aHUS patients at presentation)}

- Blood samples

- CBC, blood film, and reticulocyte count

- Coombs test, fibrinogen, and D-dimer

- Haptoglobin level, LDH

- BUN, creatinine, and full electrolytes

- Liver function tests (LFTs)

- Coagulation profile (PT, aPTT, and INR)

- C3,C4, ANA, CH 50/AH50

- Metabolic screen, serum cobalamin assay

- Blood C/S, influenza A and B screen, H1N1 screen, virology screen for HBsAg, HIV 1 and 2

- Stool for Shiga toxins (STEC) if diarrhea present

- ADAMST 13

- PCR for STEC (Shiga toxin)

- Complement factor H, I, B

- Anti-factor $\mathrm{H}$ antibodies

- C5b-9 terminal complement (MAC)

- Others

- Urine samples: (if there is urine)

- Urine dipstick (protein and blood), urine for protein/creatinine ratio

- Urine analysis and culture

B. Further laboratory investigations (after approval by the treating staff)

I. NGS (next-generation sequencing) aHUS panel in accredited genetic laboratory*

II. Then, if the result came back negative, do whole exome sequencing (WES) genetic study after genetic team consult

*Accredited laboratories where genetic studies were carried on were CGC Genetics laboratories, Mayo Medical Laboratories, Genome Diagnostics Nijmegen, and Bioscientia Human Genetics lab.

\section{Appendix 2. Eculizumab Dosing Schedule for Children with aHUS}

\begin{tabular}{lll}
\hline Pt. weight & Induction therapy & Maintenance therapy \\
\hline 5 to $<10 \mathrm{~kg}$ & $300 \mathrm{mg}$ weekly $\times 1$ dose & $300 \mathrm{mg}$ at week 2, then $300 \mathrm{mg}$ every 3 weeks \\
10 to $<20 \mathrm{~kg}$ & $600 \mathrm{mg}$ weekly $\times 1$ dose & $300 \mathrm{mg}$ at week 2, then $300 \mathrm{mg}$ every 2 weeks \\
20 to $<30 \mathrm{~kg}$ & $600 \mathrm{mg}$ weekly $\times 2$ doses & $600 \mathrm{mg}$ at week 3, then $600 \mathrm{mg}$ every 2 weeks \\
30 to $<40 \mathrm{~kg}$ & $600 \mathrm{mg}$ weekly $\times 2$ doses & $900 \mathrm{mg}$ at week 3, then $900 \mathrm{mg}$ every 2 weeks \\
$\geq 40 \mathrm{~kg}$ & $900 \mathrm{mg}$ weekly $\times 4$ doses & $1,200 \mathrm{mg}$ at week 5, then 1,200 mg every 2 weeks \\
\hline
\end{tabular}

The listed dosing regimen is from the product label $[5,11,12]$ specified by the manufacturer. 


\section{References}

1 Fakhouri F, Zuber J, Frémeaux-Bacchi V, Loirat C. Haemolytic uraemic syndrome. Lancet. 2017 Aug;390(10095): 681-96.

2 Campistol JM, Arias M, Ariceta G, Blasco M, Espinosa L, Espinosa M, et al. An update for atypical haemolytic uraemic syndrome: diagnosis and treatment. A consensus document. Nefrologia. 2015;35(5):421-47.

3 Menne J, Delmas Y, Fakhouri F, Licht C, Lommelé Å, Minetti EE, et al. Outcomes in patients with atypical hemolytic uremic syndrome treated with eculizumab in a long-term observational study. BMC Nephrol. 2019 Apr; 20(1):125-12.

4 Kavanagh D, Goodship TH, Richards A. Atypical hemolytic uremic syndrome. Semin Nephrol. 2013 Nov;33(6): 508-30.

5 Loirat C, Fakhouri F, Ariceta G, Besbas N, Bitzan M, Bjerre A, et al. An international consensus approach to the management of atypical hemolytic uremic syndrome in children. Pediatr Nephrol. 2016 Jan;31(1):15-39.

6 Fremeaux-Bacchi V, Fakhouri F, Garnier A, Bienaimé F, Dragon-Durey MA, Ngo S, et al. Genetics and outcome of atypical hemolytic uremic syndrome: a nationwide French series comparing children and adults. Clin J Am Soc Nephrol. 2013 Apr;8(4):554-62.

7 Noris M, Caprioli J, Bresin E, Mossali C, Pianetti G, Gamba S, et al. Relative role of genetic complement abnormalities in sporadic and familial aHUS and their impact on clinical phenotype. Clin J Am Soc Nephrol. 2010 Oct; 5(10):1844-59.

8 Roumenina LT, Frimat M, Miller EC, Provot F, Dragon-Durey MA, Bordereau P, et al. A prevalent C3 mutation in aHUS patients causes a direct C3 convertase gain of function. Blood. 2012 Jan;119(18):4182-91.

9 Quaggin SE. DGKE and atypical HUS. Nat Genet. 2013 May;45(5):475-6.

10 Lemaire M, Frémeaux-Bacchi V, Schaefer F, Choi M, Tang WH, Le Quintrec M, et al. Recessive mutations in DGKE cause atypical hemolytic-uremic syndrome. Nat Genet. 2013 May;45(5):531-6.

11 Raina R, Grewal MK, Burke LLG, Bagga A. Optimal management of atypical hemolytic uremic disease: challenges and solutions. Int J Nephro Renovasc Dis. 2019 Sep;12:183-204.

12 Baskin E, Gulleroglu K, Kantar A, Bayrakci U, Ozkaya O. Success of eculizumab in the treatment of atypical hemolytic uremic syndrome. Pediatr Nephrol. 2015 May;30(5):783-9.

13 US Food and Drug Administration. Soliris (eculizumab) [prescribing information]. Boston, MA: Alexion Pharmaceuticals, Inc; 2018 Jun.

14 European Medicines Agency. Soliris (eculizumab) [summary of product characteristics]. Paris: Alexion Europe SAS; 2017.

15 Legendre CM, Licht C, Muus P, Greenbaum LA, Babu S, Bedrosian C, et al. Terminal complement inhibitor eculizumab in atypical hemolytic-uremic syndrome. N Engl J Med. 2013 Jun;368(23):2169-81.

16 Licht C, Greenbaum LA, Muus P, Babu S, Bedrosian CL, Cohen DJ, et al. Efficacy and safety of eculizumab in atypical hemolytic uremic syndrome from 2-year extensions of phase 2 studies. Kidney Int. 2015 May;87(5): 1061-73.

17 Fakhouri F, Hourmant M, Campistol JM, Cataland SR, Espinosa M, Gaber AO, et al. Terminal complement inhibitor eculizumab in adult patients with atypical hemolytic uremic syndrome: a single-arm, open-label trial. Am J Kidney Dis. 2016 Jul;68(1):84-93.

18 Greenbaum LA, Fila M, Ardissino G, Al-Akash SI, Evans J, Henning P, et al. Eculizumab is a safe and effective treatment in pediatric patients with atypical hemolytic uremic syndrome. Kidney Int. 2016 Mar;89(3):70111.

19 Wong EK, Goodship TH, Kavanagh D. Complement therapy in atypical haemolytic uraemic syndrome (aHUS). Mol Immunol. 2013 Dec;56(3):199-212.

20 Sellier-Leclerc AL, Fremeaux-Bacchi V, Dragon-Durey MA, Macher MA, Niaudet P, Guest G, et al. Differential impact of complement mutations on clinical characteristics in atypical hemolytic uremic syndrome. J Am Soc Nephrol. 2007 Aug;18(8):2392-400.

21 Fujisawa M, Kato H, Yoshida Y, Usui T, Takata M, Fujimoto M, et al. Clinical characteristics and genetic backgrounds of Japanese patients with atypical hemolytic uremic syndrome. Clin Exp Nephrol. 2018 Mar;22(5): 1088-99.

22 Schaefer F, Ardissino G, Ariceta G, Fakhouri F, Scully M, Isbel N, et al. Clinical and genetic predictors of atypical hemolytic uremic syndrome phenotype and outcome. Kidney Int. 2018 Feb;94(2):408-18.

23 Raina R, Krishnappa V, Blaha T, Kann T, Hein W, Burke L, et al. Atypical hemolytic-uremic syndrome: an update on pathophysiology, diagnosis, and treatment. Ther Apher Dial. 2019 Feb;23(1):4-21.

24 Lee JM, Park YS, Lee JH, Park SJ, Shin JI, Park YH, et al. Atypical hemolytic uremic syndrome: Korean pediatric series. Pediatr Int. 2015 Jan;57(3):431-8.

25 Bu F, Borsa N, Gianluigi A, Smith RJH. Familial atypical hemolytic uremic syndrome: A review of its genetic and clinical aspects. Clin Dev Immunol. 2012;2012:370426.

26 Bresin E, Rurali E, Caprioli J, Sanchez-Corral P, Fremeaux-Bacchi V, Rodriguez de Cordoba S, et al. Combined complement gene mutations in atypical hemolytic uremic syndrome influence clinical phenotype. J Am Soc Nephrol. 2013 Mar;24(3):475-86.

27 Alzabli SM, Anazi AA, Amin M, Rahman U, Rahim KA. Complete renal recovery following delayed therapy with eculizumab in atypical hemolytic uremic syndrome. Asian J Pediatr Nephrol. 2018 Jan-Jun;1(1):33-7. 
28 Hayes W, Tschumi S, Ling SC, Feber J, Kirschfink M, Licht C. Eculizumab hepatotoxicity in pediatric aHUS. Pediatr Nephrol. 2015 May;30(5):775-81.

29 Wijnsma KL, Duineveld C, Wetzels JFM, van de Kar NCAJ. Eculizumab in atypical hemolytic uremic syndrome: strategies toward restrictive use. Pediatr Nephrol. 2019 Nov;34(11):2261-77.

30 Alfakeeh K, Azar M, Alfadhel M, Abdullah AM, Aloudah N, Alsaad KO. Rare genetic variant in the CFB gene presenting as atypical hemolytic uremic syndrome and immune complex diffuse membranoproliferative glomerulonephritis, with crescents, successfully treated with eculizumab. Pediatr Nephrol. 2017 Feb;32(5): 885-91.

31 Bienaime F, Dragon-Durey MA, Regnier CH, Nilsson SC, Kwan WH, Blouin J, et al. Mutations in components of complement influence the outcome of factor I-associated atypical hemolytic uremic syndrome. Kidney Int. 2010 Feb;77(4):339-49.

32 Lovric S, Fang H, Vega-warner V, Sadowski CE, Gee HY, Halbritter J, et al. Rapid detection of monogenic causes of childhood-onset steroid-resistant nephrotic syndrome. Clin J Am Soc Nephrol. 2014 Jun;9(6):1109-16.

33 Posey JE, Harel T, Liu P, Rosenfeld JA, James RA, Coban Akdemir ZH, et al. Resolution of disease phenotypes resulting from multilocus genomic variation. N Engl J Med. 2017 Jan;376(1):21-31.

34 Tabor HK, Auer PL, Jamal SM, Chong JX, Yu JH, Gordon AS, et al. Pathogenic variants for Mendelian and complex traits in exomes of 6,517 European and African Americans: Implications for the return of incidental results. Am J Hum Genet. 2014 Aug;95(2):183-93. http://dx.doi.org/10.1016/j.ajhg.2014.07.006. 